\title{
How to Conquer the Inconsistency of D Value Determined by Survivor Curve Method and Fraction Negative Method
}

\section{Hideharu Shintani*}

School of Science, Chuo University, Japan

According to the ISO 11138-1[1], 6.4 resistance characteristics [1], 6.4.1 resistance (D value) characteristics shall be determined by a combination of at least two of the following methods [1]:

a) Determination of the $\mathrm{D}$ (decimal reduction) value through the construction of a survivor curve

b) Determination of the $\mathrm{D}$ value through a fraction negative method

c) Verification of the survival/kill response characteristics [1].

d) Survivor curve method and

e) Fraction negative method.

Among fraction negative method, there are two major methods. One is Sperman-Karber procedure and the other is Stumbo-MurphyCochran procedure [1]. The latter is more popular as fraction negative method. Both fraction negative methods have a premise that the survivor curve from fraction negative range (as a log scale of colony count 5-10-2 $\mathrm{CFU}$, colony formation unit) to the initial population, mostly $10^{6} \mathrm{CFU}$, must be connected with straight line, indicating survivor curve method required beforehand to conduct fraction negative method.

Anyway, it is problematic that both result of $\mathrm{D}$ values from fraction negative method and survivor curve method so often differ, but as survivor curve method is more reliable, so we often choose $\mathrm{D}$ value obtained by survivor curve method. However, this procedure and decision is not correct from the requirement of ISO 11138-1.

Among fraction negative method, Sperman-Karber procedure is so complicated and the result is not reliable. Stumbo-Murphy-Cochran procedure is much easier than Sperman-Karber procedure and survival curve procedure, but D value obtained by Stumbo-Murphy-Cochran procedure is still unreliable, which is the same as Sperman-Karber procedure. This indicates that survivor curve method is most reliable and biological indicators (BI) used for survivor curve method is only 20 sheets as a whole.

When the $\mathrm{D}$ values obtained by Fraction Negative Procedure and Survivor Curve Procedure significantly differ, how would it be determined? At ISO TC (Technical Committee) 198 WG (Working Group) 4 on Biological Indicator, we discussed how to conquer this inconsistency. As Japan delegate, I insisted that resistance (D value) characteristics shall not be determined by a combination of at least two of the following methods, but either one procedure of the following methods and survivor curve procedure must be considered priority procedure because it is the most reliable procedure. This suggestion, however, was not agreed and the confused sentence was remained. I do not know how it will be conquered when survivor curve procedure and fraction negative procedure gave different $\mathrm{D}$ values in the real situation as no approved standard variation was presented. In addition, there exists inconsistency between Sperman-Karber Procedure and Stumbo-Murphy-Cochran procedure in Fraction negative procedure. The former was comparatively reliable compared with StumboMurphy-Cochran procedure, but much more complicated. So in the real situation how it will be determined when $\mathrm{D}$ vales among survivor curve method, Sperman-Karber procedure and Stumbo-MurphyCochran procedure are significantly differed. There is no description the approval variation between survival curve method and fraction negative method in ISO 11138-1, 6.4. I would like to ask the readers how do you treat the result when they significantly differed? I would like to listen the readers' opinion.

\section{References}

1. ANSI/AAMI/ISO 1138-1:2006 Sterilization of health care products- Biological Indicators- Part 1: General requirements.
*Corresponding author: Hideharu Shintani, School of Science, Chuo University, Japan, Tel: +81425922336; E-mail: shintani@mail.hinocatv.ne.jp

Received March 19, 2014; Accepted March 20, 2014; Published March 31, 2014

Citation: Shintani H (2014) How to Conquer the Inconsistency of D Value Determined by Survivor Curve Method and Fraction Negative Method. Pharmaceut Reg Affairs 3: e129. doi:10.4172/2167-7689.1000e129

Copyright: (C) 2014 Shintani H. This is an open-access article distributed under the terms of the Creative Commons Attribution License, which permits unrestricted use, distribution, and reproduction in any medium, provided the original author and source are credited. 